\title{
Infection, mimics, and autoimmune disease
}

\author{
Noel R. Rose \\ Department of Pathology and The W. Harry Feinstone Department of Molecular Microbiology and Immunology, \\ The Johns Hopkins Medical Institutions, 720 Rutland Avenue, Ross 659, Baltimore, Maryland 21205, USA. \\ Phone: (410) 955-0330; Fax: (410) 614-3548; E-mail: nrrose@jhsph.edu.
}

See related article, pages $985-993$.

The observation that infection can precipitate an autoimmune disease dates back more than a century. The first human autoimmune disease described, paroxysmal cold hemoglobulinuria, was thought of as a late consequence of syphilis, and rheumatic fever is still associated with preceding streptococcal infection. In modern times, these associations have been attributed to molecular mimicry. In its simplest form, the concept of molecular mimicry states that antigenic determinants of infectious microorganisms resemble structures in the tissues of the host but differ enough to be recognized as foreign by the host's immune system. It is now clear that mimicry on the molecular level is a common phenomenon; that is, many sequential and structural determinants of infectious agents simulate epitopes of host tissues (1). But, as Mackay and I remarked recently, "There are, as yet, no firm instances of molecular mimicry by microorganisms serving as initiating agents of human autoimmune disease..." (2).

\footnotetext{
Molecular mimicry in Chagas' disease There is probably no better candidate for investigating mimicry than the cardiomyopathy of chronic Chagas' disease. It afflicts about $30 \%$ of the 20 million individuals infected with the protozoan Trypanosoma cruzi in the Americas. The presence of a cardiac inflammatory infiltrate in apparent absence of parasites suggests that the trypanosome initiates an autoimmune response. Indeed, a number of crossreactive human antigens have been implicated by their reaction with sera of Chagas patients. They include, for example, a $23 \mathrm{kDa}$ ribosomal protein (3), a functional epitope on the $\beta_{1}$ adrenergic receptor (4), a $48 \mathrm{kDa}$ protein found in neuronal axons (5), and a heptapeptide of cardiac myosin heavy chain (6). In this issue of the JCI, Gironès and colleagues have identified another crossreactive antigen (Cha), a novel peptide from human cells (7). This peptide,
}

which reacts with the sera of patients with chronic Chagas' disease and of mice infected with T. cruzi, was found in abundance in human and mouse hearts. Cross-reaction between the mammalian and trypanosomal peptides was documented for both $T$ and $B$ cells. The finding that this peptide bears both $\mathrm{B}$ - and T-cell epitopes makes it a leading candidate for the induction of the cardiomegaly of Chagas' disease through molecular mimicry, since it would facilitate T/B-cell cooperation (8).

This work leaves critical questions unanswered. Some patients with Chagas' disease develop megacolon and megaesophagus due to destruction of parasympathetic ganglia, but Cha is not found in nervous tissue. No functional changes were associated with Cha-specific antibodies. On the other hand, mice immunized with a 13 amino acid ribosomal peptide of $T$. cruzi produced antibodies that caused functional changes in the heart without evidence of mononuclear infiltration (9). Finally, living trypanosomes induce Chagaslike lesions in the hearts of mice, but recombinant Cha does not. Thus, the question of whether molecular mimicry using a single, defined antigen of the parasite in the absence of infection actually mirrors clinical autoimmune disease remains unaddressed.

\section{Molecular mimicry abounds}

Recent insights into T-cell recognition have greatly broadened the original concept of molecular mimicry. A number of studies have shown that there is a fair measure of flexibility in the amino acid sequence acceptable for both MHC

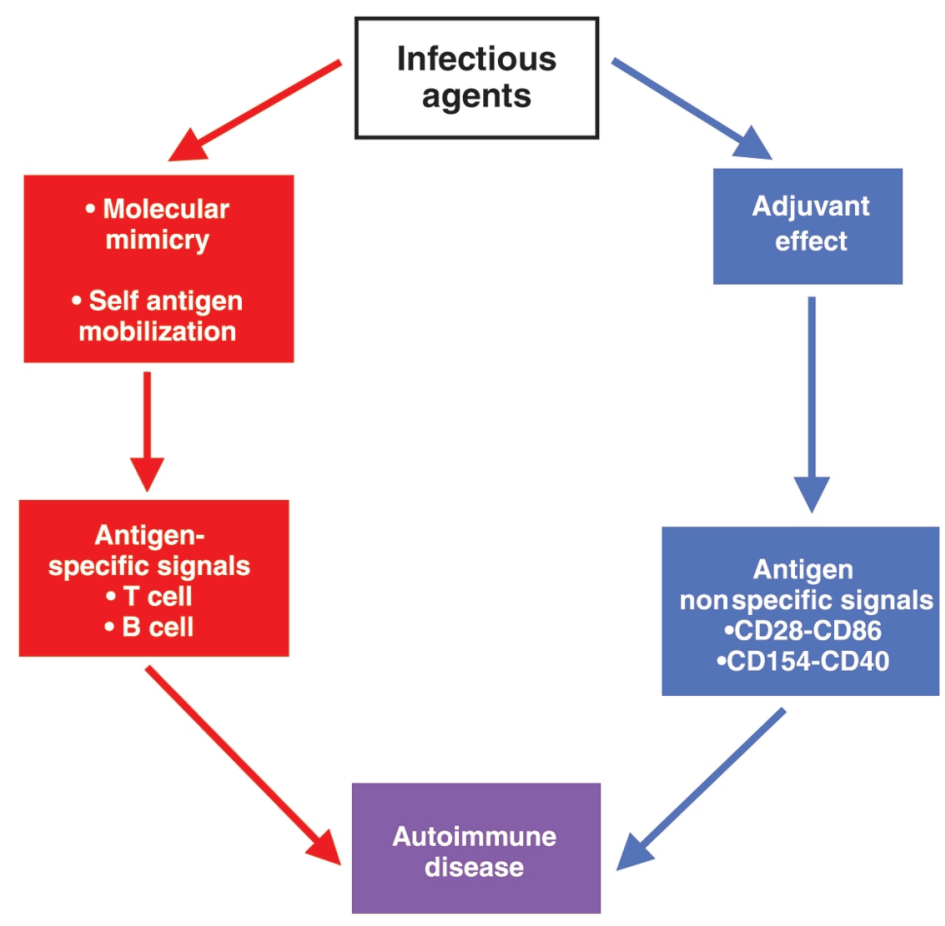

Figure 1

An infectious agent contributes to the induction of autoimmunity in two ways. First, it provides a cognate, antigen-specific signal by molecular mimicry or by mobilizing endogenous antigen (red arrows). In parallel, it causes inflammation, generating antigen nonspecific signals (blue arrows) that potentiate immune responses through what is known as an adjuvant effect. 
class II binding and for recognition by the T-cell receptor $(10,11)$. Clearly, microbial peptides with relatively limited sequence homology to myelin basic protein (MBP) can activate autoreactive T cells. Using an extensive combinatorial peptide library, Hemmer et al. (12) described differing recognition profiles of individual autoreactive T-cell clones from patients with multiple sclerosis. $\mathrm{Li}$ et al. (13) showed that, because of topological differences in their peptide finding sites, different MHC class II molecules can create different alignments of the same bound MBP peptide, thereby creating distinct $\mathrm{T}$-cell epitopes from the same peptide. Thus, T-cell recognition is even more degenerate than previously anticipated, and primary amino acid sequence similarities provide little clue to a molecular mimic.

Further expansion of the autoimmune response probably occurs due to epitope spreading. Vanderlugt and colleagues (14) developed a murine model of relapsing encephalomyelitis, using an immunodominant epitope of the myelin antigen, proteolipid protein (PLP). In the course of the disease, T-cell clones recognizing epitopes of $\mathrm{MBP}$ are detected that are not present on PLP, suggesting that the release of endogenous antigen during the initial response can stimulate self-reactive $T$ cells and play a critical role in disease progression. These data further suggest that an unrelated infection could mobilize a selfpeptide and potentiate but not initiate an attack of autoimmune disease in a subclinically primed individual (15). All of these findings demonstrate why it may be difficult to identify a single organism as the etiological agent of an autoimmune disease.

\section{The adjuvant effect}

The experiments described above remind us that the generation of an immune response depends upon two types of signals: antigen-specific recognition through the T-cell and B-cell receptors and a number of non-antigen-specific, nonclonal signals. An infectious agent may provide the specific signal by molecular mimicry or by release of endogenous antigen. The inflammatory process itself enhances the nonclonal costimulatory signals necessary to mount an immune response. Lack of appropriate costimulatory signals may account for the difference between a pathogenic and a nonpathogenic autoimmune response following infection. Our own studies with Coxsackie B3-induced (CB3-induced) myocarditis exemplify such a situation. In genetically susceptible $\mathrm{A} / \mathrm{J}$ mice, infection with CB3 induces autoimmune myocarditis (16). Injection of killed virus does not, suggesting that inflammation elicited by live virus provides costimulatory signals that influence subsequent immune responses. Evidence expanding this view came from experiments using less susceptible B10.A mice. If cotreated with bacterial LPS, as well as virus or cardiac myosin, the animals develop typical myocarditis. LPS acts through toll-like receptor 4 (TLR-4) and potently activates the innate immune response; particularly, it upregulates production of inflammatory cytokines such as IL-1 and TNF$\alpha$. Administration of either of these two cytokines converts a less susceptible to a more susceptible mouse. Furthermore, the production of autoimmune myocarditis in the susceptible mouse strain could be delayed or abrogated by administration of inhibitors of either of these two cytokines.

These results point to the importance of the inflammatory response itself in the generation of an autoimmune disease. They emphasize that an infectious microorganism may play two roles in the induction of disease (Figure 1). The first is to provide the requisite antigenic signal. This may come through molecular mimicry or through the release of excessive amounts of self-antigen from tissue cells during the infectious process. The second role of the infectious agent is to provide the adjuvant milieu in the form of upregulation of costimulatory molecules and other products of inflammation. The activation of antigen-presenting cells during microbial infection upregulates costimulatory molecules and secretion of inflammatory cytokines (17), thereby reducing the threshold needed for activation of $T$ cells by the antigenic signal. This effect, which may promote protec- tive immunity and thus benefit the host, may also prove be detrimental when it increases susceptibility to damaging autoimmune responses.

1. Oldstone, M.B.A. 1998. Molecular mimicry and immune-mediated diseases. FASEBJ. 12:1255-1265.

2. Rose, N.R., and Mackay, I.R. 2000. Molecular mimicry: a critical look at exemplary instances in human diseases. Cell. Mol. Life Sci. 57:542-551.

3. Bonfa, E., Viana, V.S.T., Barreto, A.C.P., Yoshinari, N.H., and Cossermelli, W. 1993. Autoantibodies in Chagas' disease. J. Immunol. 150:3917-3923.

4. Ferrari, I., et al. 1995. Molecular mimicry between the immunodominant ribosomal protein P0 of Try panosoma cruzi and a functional epitope on the human $\beta_{1}$-adrenergic receptor.J. Exp. Med. 182:59-65.

5. Van Voorhis, W.C., Schlekewy, L., and Trong, H.L. 1991. Molecular mimicry by Trypanosoma cruzi: the F1-160 epitope that mimics mammalian nerve can be mapped to a 12 -amino acid peptide. Proc. Natl. Acad. Sci. USA. 88:5993-5997.

6. Kalil, J., and Cunha-Neto, E. 1996. Autoimmunity in Chagas' disease cardiomyopathy: fulfilling the criteria at last? Parasitol. Today. 12:396-399.

7. Gironès, N., et al. 2001. Dominant T- and B-cell epitopes in an autoantigen linked to Chagas' disease. J. Clin. Invest. 107:985-993.

8. Liang, B., and Mamula, M.J. 2000. Molecular mimicry and the role of B lymphocytes in the processing of autoantigens. Cell. Mol. Life Sci. 57:561-568.

9. Motrán, C.C., Fretes, R.E., Cerbán, F.M., Rivarola H.W., and Vottero de Cima, E. 2000. Immunization with the C-terminal region of Trypanosoma cruzi ribosomal $\mathrm{P} 1$ and $\mathrm{P} 2$ proteins induces long term duration cross-reactive antibodies with heart functional and structural alterations in young and aged mice. Clin. Immunol. 97:89-94.

10. Hausmann, S., Martin, M., Gauthier, L., and Wucherpfennig, K.W. 1999. Structural features of autoreactive TCR that determine the degree of degeneracy in peptide recognition. J. Immunol. 162:338-344.

11. Barnaba, V., and Sinigaglia, F. 1999. Molecular mimicry and T cell-mediated autoimmune disease. J. Exp. Med. 185:1529-1531.

12. Hemmer, B., et al. 1997. Identification of high potency microbial and self ligands for a human autoreactive class II-restricted T cell clone. J. Exp. Med. 185:1651-1659.

13. Li, Y., Li. H., Martin, R., and Mariuzza, R.A. 2000 Structural basis for the binding of an immun odominant peptide from myelin basic protein in different registers by two HLA-DR2 proteins. $J$. Mol. Biol. 304:177-188.

14. Vanderlugt, C.L., et al. 2000. Pathologic role and temporal appearance of newly emerging autoepitopes in relapsing experimental autoimmune encephalomyelitis. J. Immunol. 164:670-678.

15. Rao, V.P., Kajon, A.E., Spindler, K.R., and Caray onniotis, G. 1999. Involvement of epitope mim icry in potentiation but not initiation of autoimmune disease. J. Immunol. 162:5888-5893.

16. Lane, J.R., Neumann, D.A., Lafond-Walker, A., Herskowitz, A., and Rose, N.R. 1993. Role of IL-1 and tumor necrosis factor in coxsackie virus induced autoimmune myocarditis. J. Immunol. 151:1682-1690.

17. Manickasingham, S.P., Anderton, S.M., Burkhart, C., and Wraith, D.C. 1998. Qualitative and quantitative effects of CD28/B7-mediated costimulation on naive $\mathrm{T}$ cells in vitro. J. Immunol. 161:3827-3835. 